\title{
A design of the preference acquisition detection system using the EGG
}

\author{
Hironobu Fukai ${ }^{1}$, Yohei Tomita ${ }^{2}$, Yasue Mitsukura ${ }^{3}$ \\ ${ }^{1}$ Tokyo University of Science, Chiba, Japan \\ ${ }^{2}$ Foster Electric Company, Limited, Tokyo, Japan \\ ${ }^{3}$ Keio University, Kanagawa, Japan \\ Email address: \\ fukai@rs.tus.ac.jp (H. Fukai), tomita@foster.co.jp (Y. Tomita), mitsukura@sd.keio.ac.jp (Y. Mitsukura)
}

\section{To cite this article:}

Hironobu Fukai, Yohei Tomita, Yasue Mitsukura. A Design of the Preference Acquisition Detection System Using the EEG. International Journal of Intelligent Information Systems, Vol. 2, No. 1, 2013, pp. 19-25. doi: 10.11648/j.ijiis.20130201.13

\begin{abstract}
Estimation of emotional states has been multi-disciplinary research interests. Among them, although there are many ways of the estimation such as subjective evaluations and behavioral taxonomy, direct evaluation from the human brain is more reliable. Especially, electroencephalographic (EEG) signal analysis is widely used because of its simplicity and convenience. In our research, emotional states are investigated with a simple electroencephalography which has only one electrode. This device is lighter and cheaper than existing devices.However, its feasibility has yet been proven.
\end{abstract}

Keywords: KANSEI; Human Interest; EEG

\section{Introduction}

Recently, many services that considered the personal preference have been provided. Along with it, the study of acquiring personal preference and KANSEI are actively done. KANSEI is Japanese word becausestudying at first in Japan. Generally sensitivity is inborn;it's said that KANSEI is postnatal. There are a subjective index and an objective index in the method of acquiring the preference. A subjective index is obtained by the questionnaire, and an objective index is given by the bio-signal $[1,2]$. An objective index can show the physical quantity by the numeric value. Therefore, an objective and engineered approaches possible [3]. By the way, there are a lot of reports concerning the relation between the EEG and the preference and the KANSEI in the analysis of the bio-signal [4]-[8]. This is based on the idea "the state of the brain should change if these states change because brain governs the mind, conscious, recognition and the sense", and so on $[9,10]$. Then, the EEGthat is one of the bio-signals is used for the index to acquire the preference in this study. In this study, we propose the various preference measurement systems such as for olfactory sense, acoustic sense, haptic sense, taste sense, visual sense (generally called five senses) and it's combination (i.e., acoustic sense, and visual sense). In this paper, we introduce the procedure of the EEG measurement and analysis method, and described as an example real system which is titled"Visualization of the Mind Status using the EEG". Furthermore, we demonstrate these systems by using the EEG in that day.

\section{Analysis of the EEG}

EEG is electrical signal caused by the activity of cerebral cortex. We can measure human brain activity noninvasively, high temporal resolution, and flexibility by using EEG. It is well known that EEG changes corresponding to mental condition, cerebration, and emotion. EEG measurement point is defined by the international 10-20 system. Generally, we measure the EEG based on these points using multiple-EEG having many electrodes. However, there are some problems. It takes more than 30 minutes to wear. Also, subjects need to use gel for electrolyte and that would stress them. For these reasons, multiple-electrode electroencephalograph is impractical. Therefore, we use a simple electroencephalograph which is constructed by our laboratory. This electroencephalograph is less demanding method for subjects because it is easy to wear and less stressing by nothing of tightness. Moreover, this device measures the EEG activity at Fp1(left frontal lobe) in the international $10-20$ system (sampling frequency is $128 \mathrm{~Hz}$.). There is very low noise caused by hair at this measurement point. Furthermore, the EEG changes occur in prefrontal area largely. Thus, we consider that this simple electroencephalograph is an appropriate method of measuring the EEG to estimate the 
human-interest. In addition, measurement methodology is referential recording: reference electrode is arranged at the left ear lobe and exploring electrode is at Fp1. The EEG data is analyzed by using fast Fourier transform per one sec., and we can obtain the amplitude spectra at $1-64 \mathrm{~Hz}$.

\subsection{KANSEI and Sensibility}

There are 2 ways of indicating the preferences that is, KANSEI and sensibility. These 2 words are very important. It is because preferences which are obtained in this paper are constructed by the KANSEI.Sensibility is similar to KANSEI but difference. KANSEI is Japanese words because studying at first in Japan. On the other hand, Sensibility is innate ability. KANSEI is acquired after birth. We focus on Preference from KANSEI by using EEG in this paper.

\subsection{The Simple Electroencephalograph (EEG)}

In this study, the simple electroencephalograph of the band type is used. This electroencephalograph is made by Brain Function Research \& Development Center in Japan. Fig. 2 shows this electroencephalograph. The conventional electroencephalograph (EEG) is expensive, large, and impossible to measure under in a natural environment. Therefore, the subjects are strained.This electroencephalograph is compactly made as $120 \mathrm{~mm}(\mathrm{~W}), 135 \mathrm{~mm}(\mathrm{D})$, and $35 \mathrm{~mm}(\mathrm{H})$ and can be measured under the practical environment. Therefore, it's less burdensome on subjects. The electrode is fixed to the headband. The measurement position is electrode arrangement Fp1 in international 10-20 system. Fig. 1 shows the international 10-20 system. We can obtain the discrete time data that the EEG of one second is analyzed frequency to $24 \mathrm{~Hz}$ at $1 \mathrm{~Hz}$ interval. The band filter from 4 $\mathrm{Hz}$ to $22 \mathrm{~Hz}$ is used for this simple electroencephalograph. Therefore, we use the time series data of each frequency components between 4 and $22 \mathrm{~Hz}$.

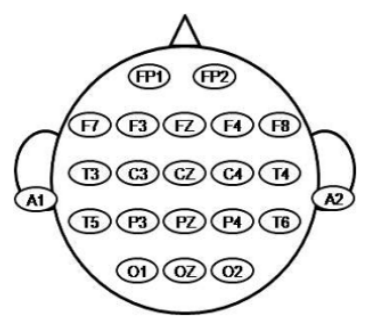

Figure 1. The points from international 10-20 method.

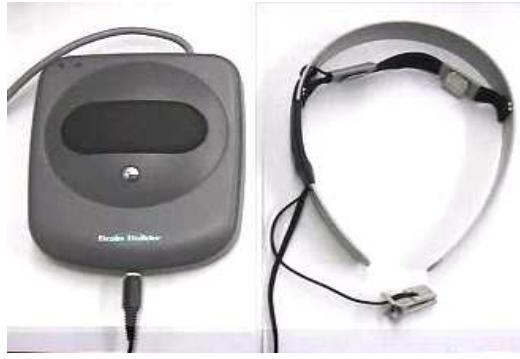

Figure 2. Our proposed EEG device.
The EEG devices in Fig. 3 are conventional EEG devices. In these devices, it is a long time to wear. The left one, it is 45 minutes and the right one needs about 30 minutes, respectively. On the other hand, in our EEG device, it takes 30 seconds to wear.This device is easy to wear and we can reduce of the number of electrodes. The contribution of our devices is as follows:

Reduction of the number of electrodes

Needless of gel for the wearing

Easily wearing by oneself every time and everywhere
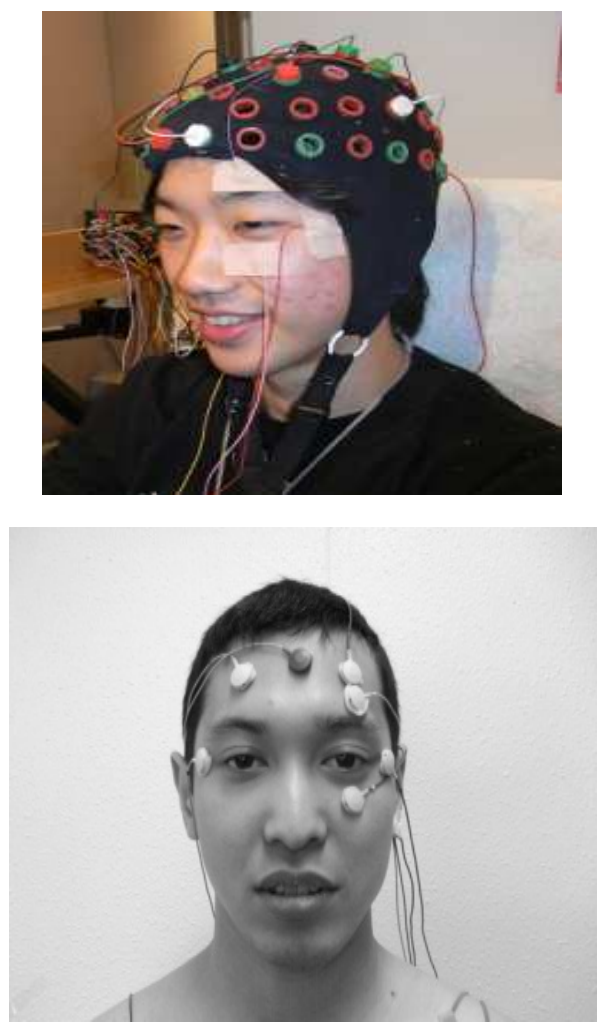

Figure 3. Conventional EEG device.

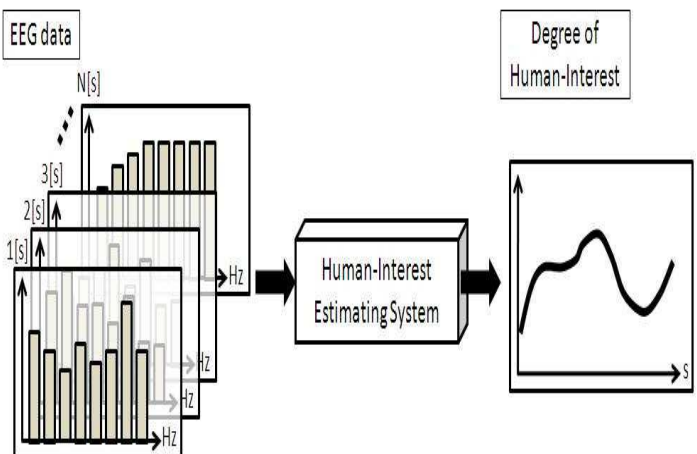

Figure 4. The Principle of the proposed method2.3.Investigation of Preferences.

In particular, we usually used questionnaire as a preference investigation method, It is easy to understand preference but difficult to obtain sequential measurement [11]. We need the sequential investigation in this paper. Therefore, we pay attention to brain activity which is the solution to 
problem point.

\subsection{Pre-processing for the Noise, Data Mining, andPat- tern Recognition}

The method is FA [12], ICA, PCA, Multiple-regression, and so on. Then, the data which is analyzed by pre-processing, the stochastic method such as Incremental PCA, FLDA, SPCA, AMUSE, SFLDA is used for data mining. Then, pattern recognition method is applied for the system we want. In these flow(Fig. 4), which methods we use is determined by the GA.

\section{Experiment 1}

\subsection{Estimation System for Human-Interest Degree}

In this section, we explain the estimation system for human-interest degree in detail. First, we show the system summary. Then, we describe the method of constructing our system. In particular, we explain the method of EEG measurement and feature extraction, an EEG clustering, and a multiple regression analysis.

Fig. 5shows the overview of estimation system for human-interest degree. Our system estimates the human-interest degree while watching TV commercials sequentially.First of all, weacquire the EEG data while watching TV commercials using the simple electroencephalograph. Moreover, we apply the EEG data to our system. After that, we get the sequential estimation result for human-interest degree.

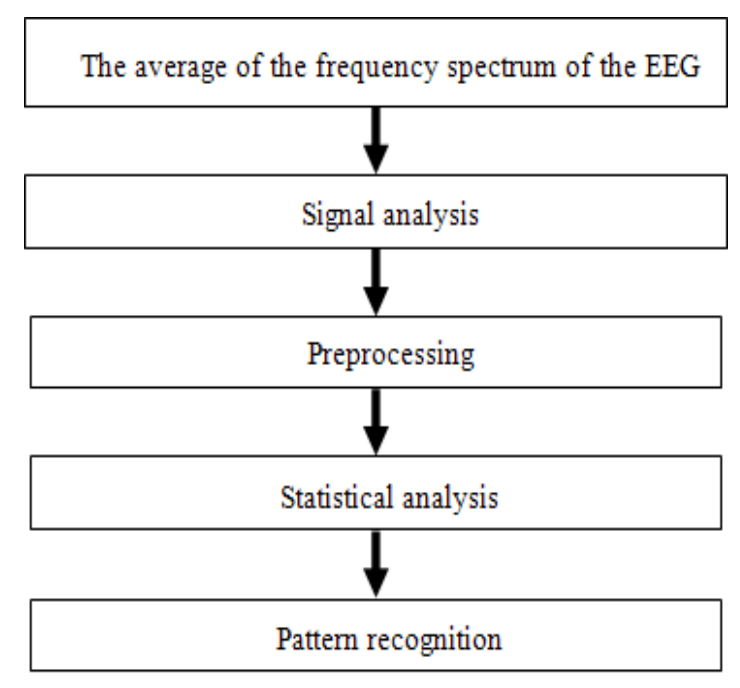

Figure5. The Procedure of the proposed method.

\section{Construction of Estimation system for Human-Interest Degree}

First of all, we show about the EEG features and the questionnaire. Then, we classify the subjects by the EEG feature using a cluster analysis because we consider the individual characteristic of the EEG [13]. We explain the cluster analysis. Finally, in order to estimate the human-interest degree, we apply the EEG features andthe result ofquestionnaire to a multiple regression analysis. We show the multiple regression analysis.

Questionnaire The questionnaire shows human's emotion or cerebration by linguistic expression. Moreover, this is called subjective assessment. In this experiment, the subjects evaluate the human-interest degree for each TV commercial on a scale of zero to ten. We use this degree when we analysis the human-interest.

EEG features The EEG features are the EEG data of amplitude spectra of 4-22Hz. In this experiment, we suppose that the EEG features show the change of human's emotion or cerebration while watching TV commercials. In addition, when we construct the system, we use the time average of the spectrum while watching TV commercials.

Cluster Analysis The cluster analysis is used for clustering the subjects using the individual characteristic of EEG. In particular, we apply the hierarchical algorithm. We begin with each data and proceed to divide it into successively smaller clusters. Moreover, the distance function is important factor for classification. There are some distance functions however we adopt the Euclidean distance because this distance is most common method. In this paper, we classify into the subjects depending on the EEG features by the cluster analysis. Furthermore, we decide the effective cluster number by investigation the relationship between the number of data and the number of variable (EEG features)[14].

Multiple Regression Analysis We use the multiple regression analysis to quantitatively evaluate the degree of human-interest. The multiple regression analysis is one of the multivariate analysis to have been constructed the estimation model from a set of training data. The multiple regression equation as estimation model is constructed by using some explanatory variables and an objective variable.In this paper, we apply the EEG features as the explanatory variables and the result of questionnaire as the objective variable. That is, we estimate the result of questionnaire using the EEG features.

\section{Experiment of Watching TVCom- mercials}

In this section, we show about the experiment of watching TV commercials. We measured the EEG while watching each TV commercial and investigated the degree of human-interest for each TV commercial using questionnaires. In this experiment, the subjects are set up as 54 people, including 28 males and 26 females. Each subject watches 10 different kinds of TV commercials that consist of 15 seconds. We measure the EEG while watching TV commercials using the simple electroencephalograph. After watching each TV commercial, we investigate the degree of human-interest for each TV commercial using questionnaires. Then, Fig. 6 shows the experimental procedure.Moreover, TABLE 1 represents the experimental condition and data.Furthermore, 
TABLE 2 is used for the questionnaires in this experiment.

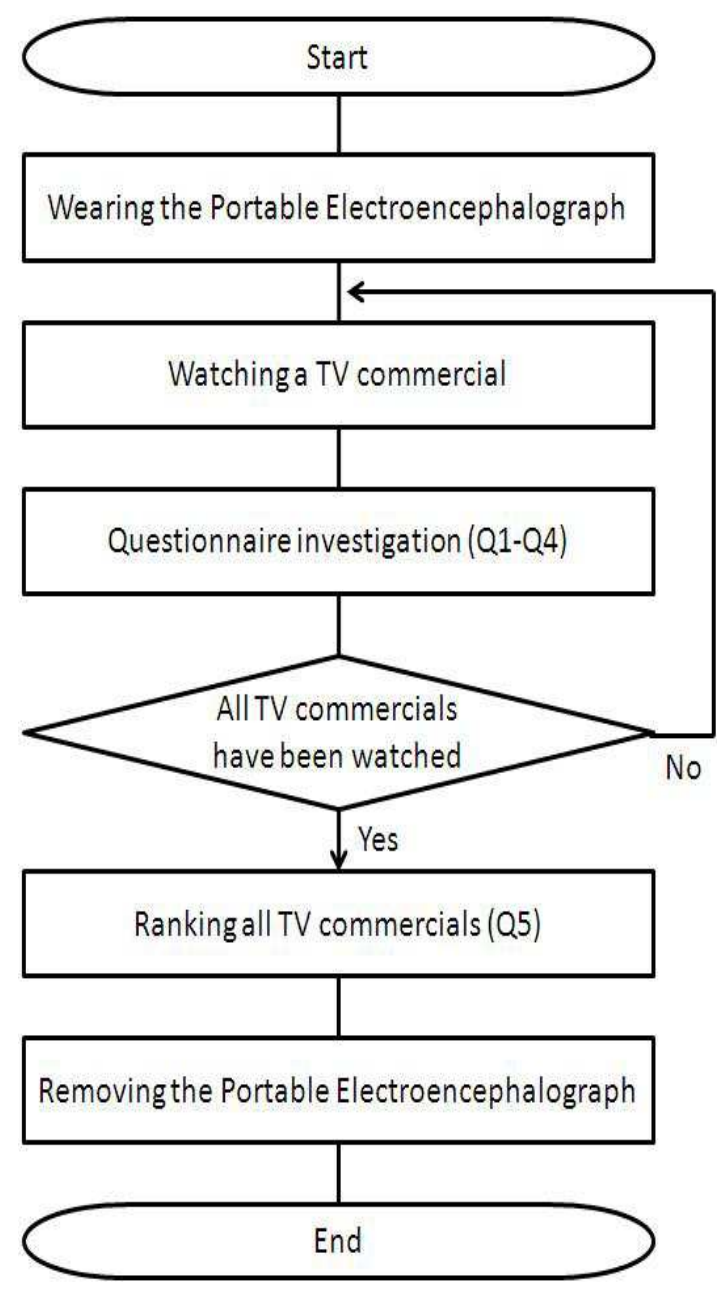

Figure 6. Experimental Procedure

Table 1. Experimental Condition and Data

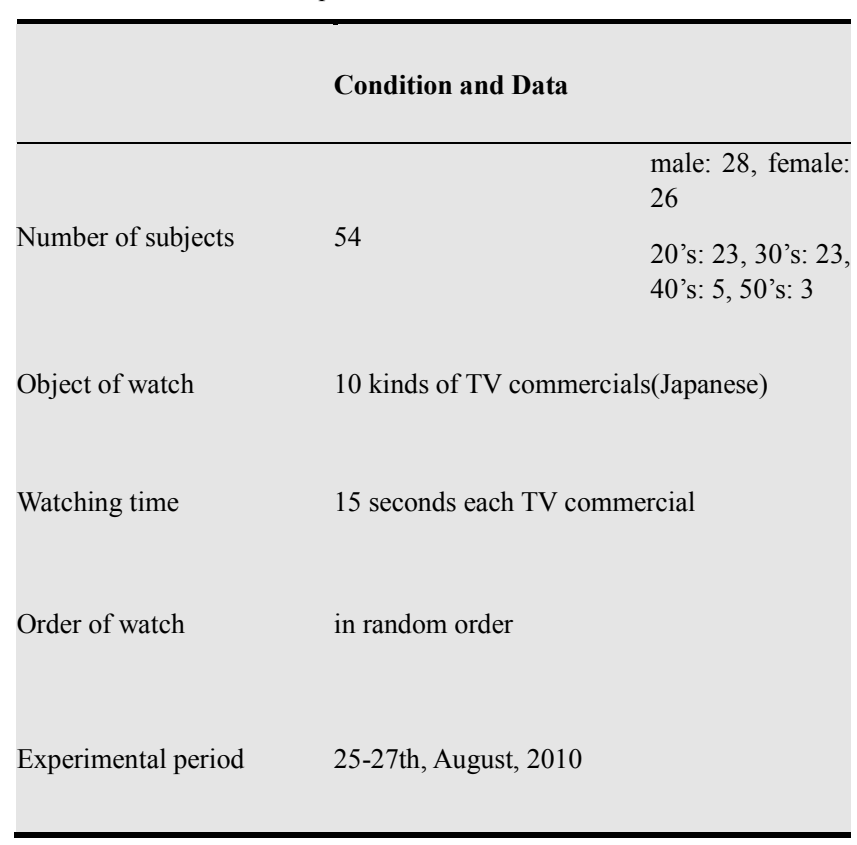

Table 2. The Questionnaire Items

\begin{tabular}{ll}
\hline Number & Questions \\
\hline Q1 & $\begin{array}{l}\text { How do you rate your interest degree } \\
\text { for the TV commercial on a scale of } 0 \text { to } 10 ?\end{array}$ \\
& $\quad \begin{array}{l}\text { Which scenes are you interested in? } \\
\text { Q2 }\end{array}$ \\
Q3 & $\begin{array}{l}\text { How many times have you seen the TV commercial? } \\
\text { (Choose i. 0, ii. 1-3, iii. 4- ) }\end{array}$ \\
& $\begin{array}{l}\text { How many times have you use/touch } \\
\text { the products/services? } \\
\text { (Choose i. 0, ii. 1-3, iii. 4- ) }\end{array}$ \\
& $\begin{array}{l}\text { Rank TV commercials } \\
\text { in descending order of human-interest degree. }\end{array}$ \\
&
\end{tabular}

\subsection{Simulations}

We explain the results of simulations for evaluation of our system. We conducted two kinds of simulations in order to discuss the effectiveness of our system. The first is the estimation of human-interest degree for each TV commercial. The second is the sequential estimation of human-interest degree for TV commercials. We describe the each result.We verify the effectiveness and generalization of the estimation system, particularly about the subject clustering with the EEG features. In order to evaluate our system, we define accuracyrate [\%] as follows:

$$
\text { accuracy } \text { rate }[\%]=\frac{\text { CORRECT }}{\text { TOTAL }} \times 100
$$

We use the most high-scoring and the most low-scoring TV commercials by the questionnaire(Q2) to calculate accuracy rate[\%]. Then, we define the human-interest degree which is estimated using the EEG by A and the score of questionnaires by $\mathrm{B}$. We determine the situation which the higher TV commercial of A matches higher one of B by CORRECT. In addition, TOTAL indicates the number of all data.

Table 3. The Number of Cluster and Accuracy Rate

\begin{tabular}{ll}
\hline Number of Cluster & Accuracy Rate[\%] \\
\hline 1 & 74.1 \\
2 & 79.6 \\
3 & 74.1 \\
4 & 75.9 \\
\hline
\end{tabular}


Incidentally, Table 3 shows the relationship between the number of subject cluster and the accuracies of ranking of 2 TV commercials by proposed system.According to Table 3 , we find that 2 clusters is the highest accuracy rate. In contrast, we considered that more number of clusters was higher accuracy rate because the number of data and variance of datawere smaller. However, in this simulation, we regard that we classify the subjects to 2 clusters because accuracy rate is not proportional to the number of cluster. Furthermore, we show the effectiveness of estimation of human-interest degree for each TV commercial by the result of accuracy rate $79.6 \%$.

\subsection{Sequential Estimation of Human-Interest Degree for TV Commercials}

We attempt to estimate the human-interest degree for TV commercials sequentially. In previous section, we show the effectiveness of our estimation system. Accordingly, in order to estimate human-interest degree sequentially, we use that system. When we measure the EEG using the simple electroencephalograph, the EEG signals of 1 second are transformed into frequency components. For this reason, we estimate the human-interest degree for TV commercials every1 second. However, we apply moving average to the EEG features because we need consideration perception time, remainder time, and measuring error. Fig. 7. shows the example of sequential estimation. Based on the graph of Fig. 7. (a) or (c), we confirm an upward tendency around the interest scene from the questionnaire. Moreover, there are some subjects which the estimation results have a few ups and downs, (see Fig. 7. (b)). Furthermore, according to the graph of Fig. 7. (d), there are a lot of scenes which the subject are interested in by the questionnaire. On the other hand, human-interest degree by the estimating system has a lot of ups and downs. According to these results, we consider that we show the fluctuation of human-interest degree which we cannot understand human-interest by the questionnaire. Furthermore, we found the personal difference of the questionnaire.

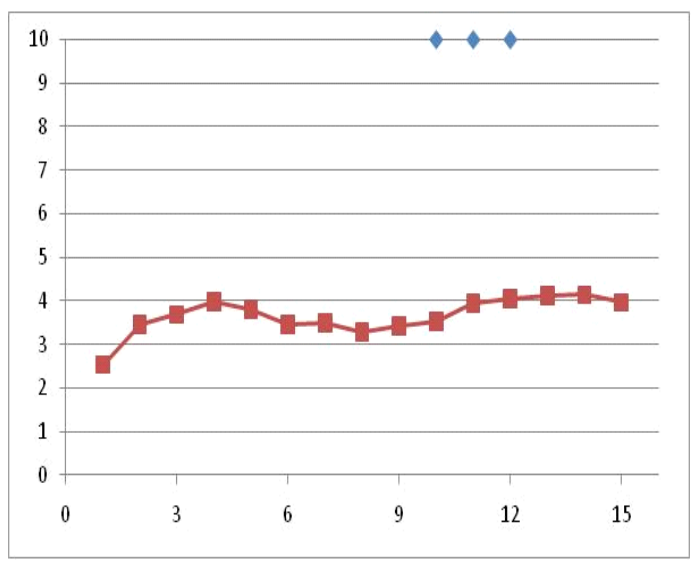

(a) Subject1/CM7

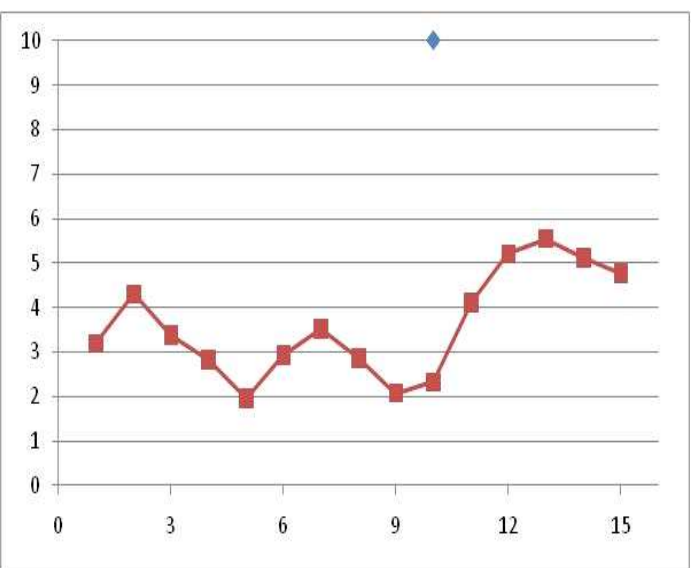

(b) Subject13/CM1

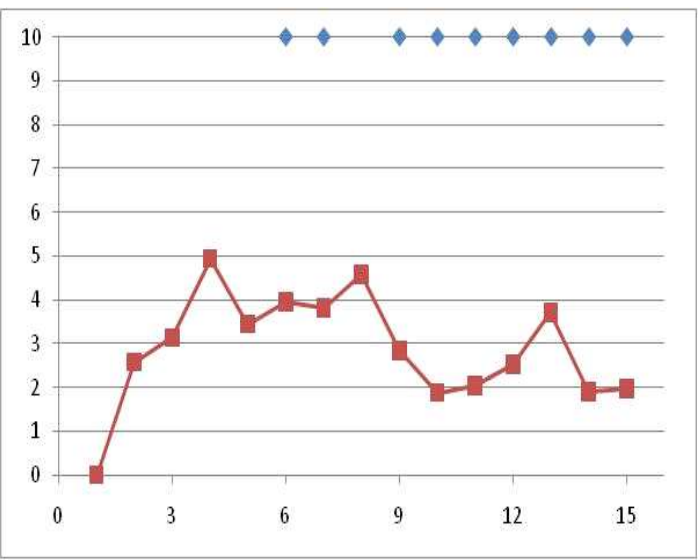

(c) Subject34/CM4

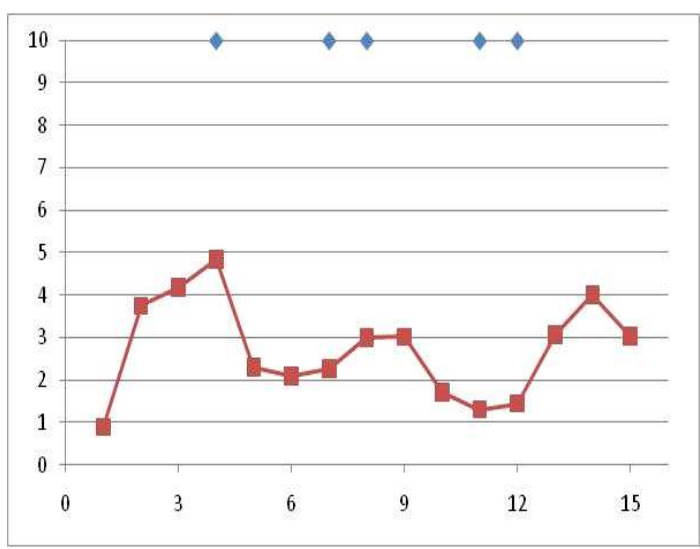

(d) Subject43/CM8

४:Human-Interest from Questionnaire $-1-0$ - Human-Interest Degree from Estimating System

The vertical axis'. Human-interest degree obtained by the proposed system. The horizontal axis: The time[second]

Figure 7. Results by the Proposed System.

\subsection{Visualization of the Mind Status using the EEG}

The rules we detected by this study are as follows: As for haptic sense, the combination of 6 and $10 \mathrm{~Hz}$ is related by the favorite. On the contrary, the combination of 11 and 16 
$\mathrm{Hz}$ indicate the dislikeable. In the olfactory sense,favorite indicate in the combination of the 4 and $7 \mathrm{~Hz}$, dislikeable put in an appearance at 9 and $14 \mathrm{~Hz}$ and so on. Furthermore, the dozing is related by the 5,6,9, and $16 \mathrm{~Hz}$. In these ways, we lead various preference measurement systems(Fig. 8.).

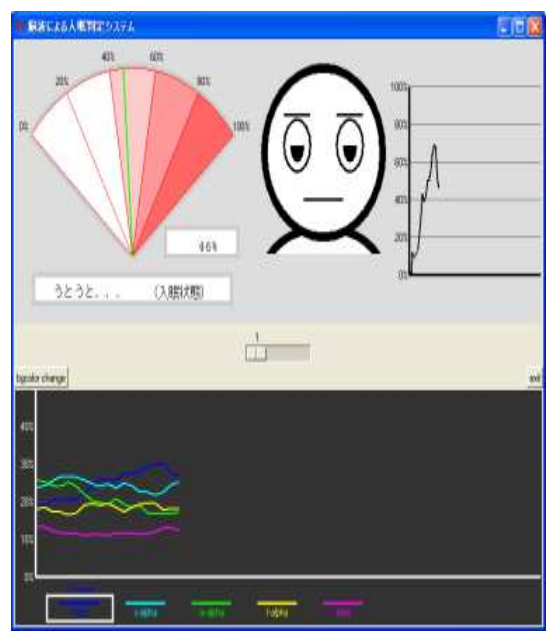

(a)

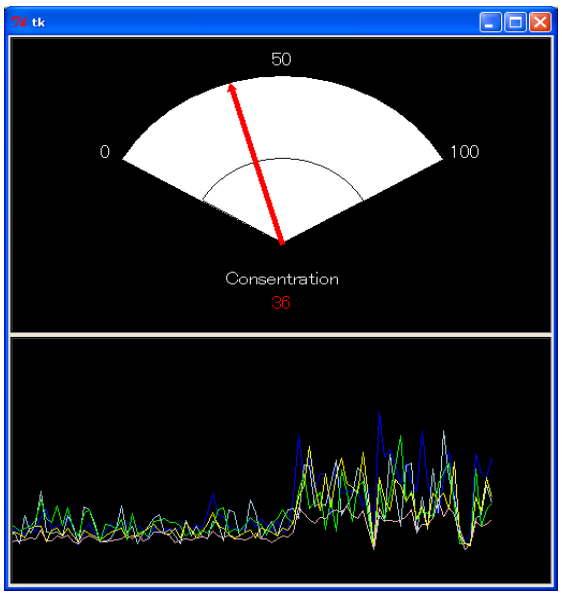

(b)

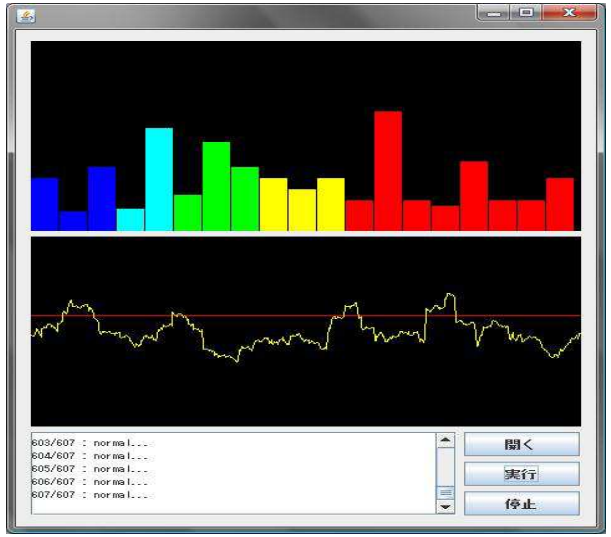

(c)

Figure 8. (a) Dozing detection system (b) Concentration degree detection system (c) Stress visualization.

\section{Conclusions}

In this study, we proposed the new preference measurement systems for five senses by using the EEG detection device which is constructed by our laboratory. In our system, the importance of frequencies and these combinations are obtained. Moreover we structured the application for these evaluations in the ubiquitous environment.

This paper shows the feasibility of our device. Additionally, further investigations are to be required with respect to a signal noise ratio and repeatability of the emotional estimation.

\section{References}

[1] K. Nagamine, A. Nozawa, and H. Ide, "Evaluation of emotions by nasal skin temperature on auditory stimulus and olfactory stimulus", IEEJ Trans. EIS, Vol. 124, No. 9, pp. 1914-1915 (2004).

[2] J. Han, and A. Uchiyama, "The measurement and analysis on the effect of olfactory stimulus on the human body", IEE Japan, Vol. 122-C, No. 9, pp. 1616-1623 (2002).

[3] D., M. W. Bos, L. F. Nordgren, and R. B. van Baaren: "On Making the Right Choice: The Delibera-tion-Without-Attention Effect", Science, Vol. 311, No. 5763, pp. 1005-1007 (2006).

[4] K. Takahashi, "Remarks on Emotion Recognition using Brain-Body Actuated System -Emotion classification using support vector machines", Prof. of Japan Mechanic Sympo-sium, pp.02-10 (2002).

[5] H. Tagaito, "An Evaluation of User Interface with Emotions", UNISYS TECHNOLOGY REVIEW, No.64, pp.103-114 (2000).

[6] S. Ota, S. Ito, Y. Mitsukura, and M. Fukumi, "Proposal for the extraction method of personal comfort and preference by the EEG maps", SICE-ICASE International Joint Conference, pp. 604-607 (2006).

[7] M. Funada, M. Shibukawa, and S. Ninomija, "Objective measurement of event related potentials' changes", The Japanese Journal of Ergonomics, Vol. 38, pp. 538-539 (2002).

[8] S. Ayabe, "Nurtured odor preference", J.Japan Associ-ation on Odor Environment, Vol. 35, No. 2, pp. 68-70 (2004)

[9] R. Ohme, M. Matuskin, and T. Szczurko: "Neurophy-siology uncovers secrets of TV commercials", DER MARKT, Vol. 49, No. 3-4, pp. 133-142 (2010).

[10] G. Vecchiato, L. Astolfi, F. D. V. Fallani, F. Cincotti, D. Mattia, S. Salinari, R. Soranzo, and F. Babiloni: "Changes in Brain Activity During the Observation of TV Commercials by Using EEG, GSR and HR Measurements", BRAIN TO-POGRAPHY, Vol. 23, No. 2, pp. 165-179 (2010).

[11] S. Tasaki, T. Igarashi, N. Murayama, and H. Koga, "Relationship between biological signals and subjective es-timation while humans listen to sounds", T.IEE Japan, Vol. 122-C, No. 9, pp. 1632-1638 (2002). 
[12] S. Shiba, Factor Analysis Method, University of Tokyo Press, (1976).

[13] S.Ito, Y. Mitsukura, M. Fukumi, and N. Akamatsu, "Proposal of the EEG analysis method using the individual characteristic of the EEG", T.IEE Japan, Vol. 124-C, No. 64, pp.1259-1266 (2004).

[14] F. Lotte, M. Congedo, A. Lecuyer, F. Lamarche, and B. Arnaldi: "A review of classification algorithms for EEG-based brain-computer interfaces", Journal of NeuralEngineering, Vol. 4, No. 2, pp. R1-R13 (2007). 